\title{
Effect of Ethanol Deprivation and Re-Exposure on the Ethanol Drinking Behaviour of the High-Alcohol-Drinker (UChB) Rats
}

\author{
Lutske Tampier, Maria Elena Quintanilla \\ Molecular and Clinical Pharmacology Program, Institute of Biomedical Sciences, Faculty of Medicine, \\ University of Chile, Santiago, Chile \\ E-mail: Itampier@med.uchile.cl
}

Received January 4, 2011; revised February 20, 2011; accepted February 22, 2011

\begin{abstract}
Alcohol addiction constitutes a major health problem in the general population, it is a complex pathology characterized by the development of tolerance, physical dependence and compulsive ethanol-seeking behaviour that often manifests as a chronic relapsing syndrome. One of the major concerns in the treatment of alcohol-dependent patients is the prevention of relapse during periods of abstinence. The alcohol deprivation effect (ADE) is defined as a temporary increase in the voluntary intake of ethanol when it is reinstated after a period of alcohol deprivation and has been used as an animal model of relapse-like drinking. ADE can be used to evaluate the efficacy of possible pharmacological agents to prevent relapse drinking. The current study was undertaken to examine whether the high-alcohol-drinker UChB rats would display an ADE. Rats were given either continuous or periodic concurrent access to $10 \%, 20 \%$ (vol/vol) of ethanol across deprivation cycles. UChB rats consuming ethanol voluntarily for two months, exhibit a robust ADE after a single deprivation period of two weeks. The increased alcohol intake during the early days of re-exposure, following a withdrawal phase, is attributed to a shift in preference towards the higher concentration of ethanol that might reflect an increase in craving for alcohol. Since an ADE is also observed in UChB rats, make this line of rats selectively bred for their high voluntary ethanol consumption, a useful model for study the efficacy of pharmacological agents for the treatment of relapse of alcohol consumption.
\end{abstract}

Keywords: Alcohol Intake, Alcohol Deprivation, Alcohol Drinking Rats, UChB Rats

\section{Introduction}

Addiction can be best defined as the loss of control over drug use. It is caused by the action of a drug of abuse and generally requires repeated drug exposure. One of the drugs more abused is alcohol. Alcohol addiction (Alcoholism) constitutes a major health problem in the general population, it is a complex pathology characterized by the development of tolerance, physical dependence and compulsive ethanol-seeking behaviour that often manifests as a chronic relapsing syndrome. As a matter of fact, one of the major concerns in the treatment of alcohol-dependent patients is the prevention of relapse during periods of abstinence.

According to Spanagel and Hölter (1999) [1], alcohol drinking over a long period of time can be separated into three stages. First, the initiation or acquisition of alcohol drinking, followed by a second stage of controlled alcohol-drinking behaviour and eventually a third stage where uncontrolled alcohol-drinking behaviour occurs, leading to the development of dependence, which has been characterized as an progressive dysregulation of brain reward and stress circuits [2,3]. Although these various stages of alcohol drinking occur in humans, it is not clear whether those transitions in alcohol drinking from one stage to another can also be observed in all animals with a high preference and a high daily intake of alcohol Various approaches have been used to evaluate the occurrence of addictive behaviour in laboratory animals; most of them based on changes in the amount of alcohol intake following a period of abstinence and a shift of preference towards higher concentration of ethanol solutions [1]. The alcohol deprivation effect (ADE) has been proposed as a model of "loss of control" and 
"relapse-like" drinking [4,5] and that can be used to evaluate the efficacy of possible pharmacological agents to prevent relapse drinking. ADE is defined as a temporary increase in the ethanol preference and in the voluntary intake of ethanol solutions over baseline drinking conditions, when alcohol is reinstated after a period of abstinence [6]. The negative consequences of withdrawal, trigger further drinking and has been hypothesized to be an animal model of alcohol craving [5]. An ADE and the modification of ethanol intake by exposure to repeated cycles of alcohol availability and deprivation has been demonstrated in the selectively bred alcohol-preferring (P) and high-alcohol-drinking (HAD) line of rats $[4,7,8]$ but not in Sardinian alcohol preferring (sP) rats [9]. However the effects of repeated deprivations or access to different ethanol concentrations have not been tested in the high-alcohol-drinking (UChB) rats, selectively bred in this laboratory for high voluntary ethanol consumption using the two-bottle choice (10\% v/v ethanol and water) paradigm $[10,11]$. The UChB rats slowly increased their intake of ethanol over 20 days in the free choice condition to a steady state point reaching 7-9 g ethanol per kg body weight per day. The gradual increase in the ethanol consumption displayed by the UChB rats across the time appeared to be the results of neuroadaptative changes subsequent to chronic intake. The objective of this study is to examine whether $\mathrm{UChB}$ rats would display an ADE and a shift in ethanol concentration preference from lower concentrations (10\%) to higher concentration (20\%) of ethanol across deprivation cycles.

\section{Animals and Methods}

\subsection{Animals}

The animals were female UChB rats from the $82^{\text {nd }}$ generation (bred at School of Medicine, University of Chile). At three months old, rats were housed individually in cages where they received concurrent free access to $10 \%$ $(\mathrm{v} / \mathrm{v}), 20 \%(\mathrm{v} / \mathrm{v})$ ethanol and water and solid food ad libitum for 8 weeks Rats were maintained on a 12-hr reversed light/dark cycle (lights off at $19.00 \mathrm{hr}$ ). During the duration of testing, animals were weighted weekly. All procedures used in this study were in compliance with the Chilean Ethical animals rules.

\subsection{Experimental Procedure}

After the period of 8 weeks under the concurrent free choice condition between 10\%, 20\% ethanol and water paradigm, nine animals were deprived from ethanol for 15 consecutives days (alcohol deprivation period). After the first deprivation period, rats received a continuous free-choice access to both ethanol solutions $(10 \%, 20 \%)$ and water for an additional two-week period and ethanol and water consumption recorded every 24 hours $\left(1^{\text {st }}\right.$ ADE). After these 2 weeks of ethanol re-exposure, rats were again deprived of ethanol for 2 additional weeks, followed by 2 weeks of ethanol exposure $\left(2^{\text {nd }} A D E\right)$. This cycle was again repeated ( $\left.3^{\text {rd }} \mathrm{ADE}\right)$. Results are expressed as grams of total ethanol intake related to kgbody weight per day, and as grams of ethanol of each ethanol solution ( $10 \%$ or $20 \%$ ) consumed per kg- body weight per day. Total water intake (water present in ethanol solution plus water from the water tube) was expressed as $\mathrm{ml} / \mathrm{kg} /$ day). Base data were the average of 7 days immediately before deprivation. An additional group of nine rats was never deprived of ethanol and served as non deprived controls and their consumption were evaluated at 8 weeks and at 18 weeks of continuous ethanol consumption.

\subsection{Statistical Analysis}

Data is expressed as the mean \pm SEM of each condition. To determine whether consumption of total ethanol or for a particular ethanol solution was increased after ethanol re-exposure, individual repeated measures ANOVA were performed separately on each of the deprivation groups and compared to the base data of the non deprived group. All post hoc comparisons were performed by using the Neuman-Keuls test. $\mathrm{P}<0.05$ was considered statistically significant.

\section{Results}

\section{Effect of Deprivation on Post-Daily Ethanol In- take by UChB Rats}

After the first deprivation period ( $1^{\text {st }} \mathrm{ADE}$ ), UChB rats displayed a significant increase in the total amount of ethanol consumed during ethanol re-exposure (Figure 1). Studies over a period of 24 hours a day showed a significant increase in ethanol intake $[(\mathrm{F}(6,69)=8.55$; $\mathrm{p}<$ 001)]. A post hoc analysis revealed that the mean daily ethanol intake of the rats was significantly higher during the initial 2 days after re-exposure, compared to base ethanol intake. Also after the second deprivation period $\left(2^{\text {nd }} \mathrm{ADE}\right)$ and third deprivation period $\left(3^{\text {rd }} \mathrm{ADE}\right)$ rats significantly increased their total ethanol consumption after ethanol re-exposure $[(\mathrm{F} 6,69)=9.74 ; \mathrm{p}<0.001]$ and $[(F(6,69)=7.21 ; p<0.001]$ respectively (Figure 1). The post hoc analysis revealed a significant increase of ethanol intake following the first 2 days of re-exposure (Figure 1). No significant statistical difference in the total ethanol intake, analyzed by the one way ANOVA $\left[(\mathrm{F}(3,165)=0.3]\right.$ was observed between $1^{\text {st }}, 2^{\text {nd }}$ or $3^{\text {rd }}$ $\mathrm{ADE}$ (Figure 1). Each time ethanol intake returns to the 
baseline around $7.5 \mathrm{~g} / \mathrm{kg} /$ day. Total water intake (sum of water from ethanol solution plus water from the water tube) about 100 to $120 \mathrm{ml} / \mathrm{kg}$ body weight/day, remains stable during the entire experimental period.

The patterns of the two ethanol solutions, consumed during 24 hours of consumption are depicted in Figure 2. The intake of $20 \%$ ethanol increased after the ethanol re-exposure $[(\mathrm{F}(6,69)=3.62 ; \mathrm{p}<0.005)]$. The post hoc analysis revealed that the amount of $20 \%$ ethanol solution increased during the initial 2 days of ethanol re-exposure. Whereas, the amount of the $10 \%$ ethanol solution did not show a significant difference $[(F 6,69)=2.8 ; n . s]$. The preference for the $10 \%$ ethanol solution remains stable. The increase in ethanol consumption after the $2^{\text {nd }}$ $\mathrm{ADE}$ and $3^{\text {rd }} \mathrm{ADE}$ was also due to a shift in the preference for the more concentrated $20 \%$ ethanol solution $[(\mathrm{F} 6,69)=6.94 ; \mathrm{p}<0.001]$ and $[(\mathrm{F} 6,69)=4.64 ; \mathrm{p}<$ $0.001)]$ respectively. The post hoc analysis revealed that the intake of the $20 \%$ ethanol solution is significantly higher during the first two days of ethanol re-exposure (Figure 2). Rats show a preference for the more concentrated ethanol solution. Overall there was a significant increase in ethanol intake and an effect of ethanol concentrations.

The total ethanol consumption and the relative intakes of the 10 and $20 \%$ ethanol solutions and water, in the non deprived UChB rats, under a 24-hr free-choice conditions appears in Table 1. Each value is the mean of the last seven consecutive days of the period of 8-weeks of

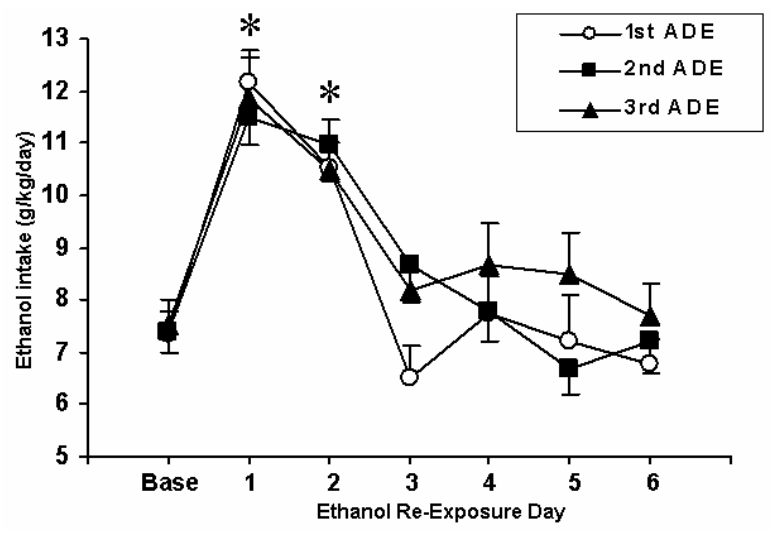

*Significantly higher than base data.

Figure 1. The mean $( \pm S E M)$ total amount of ethanol consumed (g/kg/day) by UChB rats under $24-\mathrm{hr}$ free-choice condition, deprived of ethanol for 2 weeks and then subjected to one to three cycles of 2 weeks of ethanol drinking and 2 weeks of deprivation $\left(1^{\text {st }}, 2^{\text {nd }}\right.$ and $3^{\text {rd }}$ ADE).

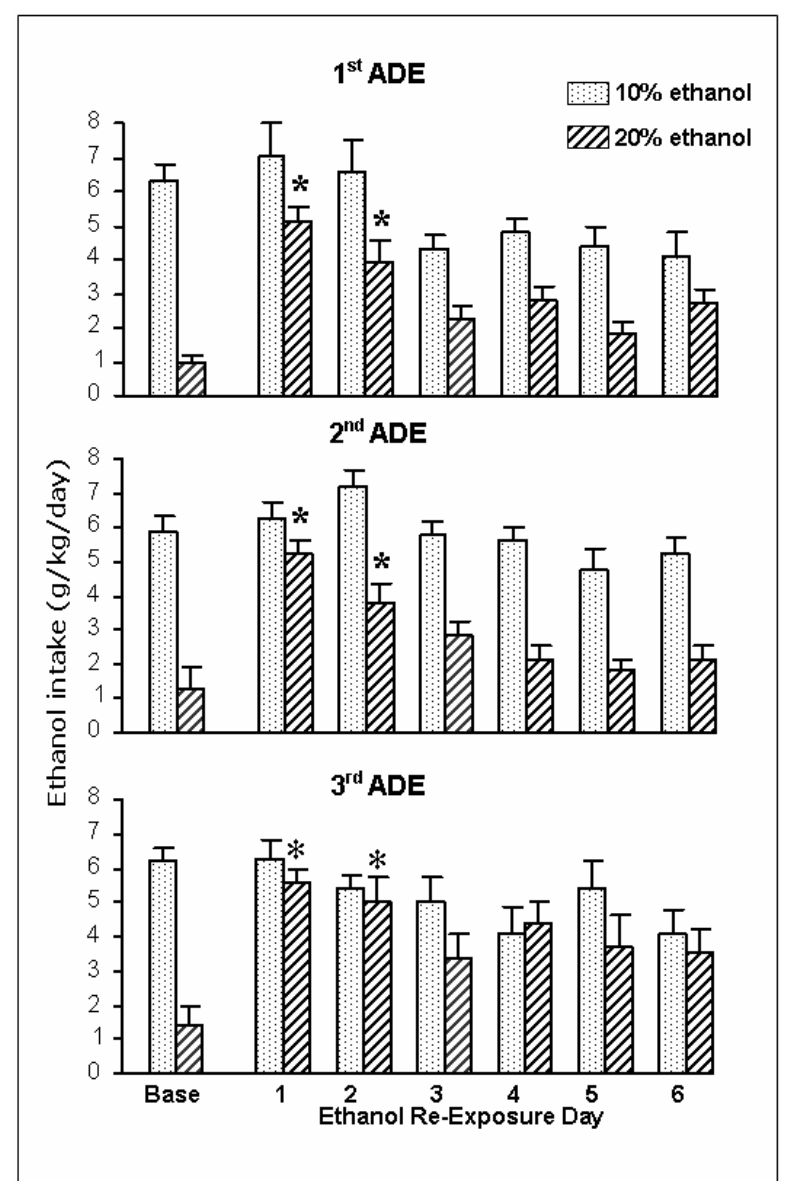

*Significantly higher than the base data of the $20 \%$ ethanol solution.

Figure 2 . The mean ( \pm SEM) of ethanol $10 \%$ and $20 \%$ consumed (g/kg/day) by UChB rats under $24-\mathrm{hr}$ free-choice condition, deprived of ethanol for 2 weeks and then subjected to one to three cycles of 2 weeks of ethanol drinking and 2 weeks of deprivation $\left(1^{\text {st }}, 2^{\text {nd }}\right.$ and $3^{\text {rd }}$ ADE)

Table 1. Ethanol, water and total water intake in non deprived UChB controls rats at two periods of continuous access to a $10 \%, 20 \% \mathrm{v} / \mathrm{v}$ ethanol solution and water.

\begin{tabular}{ccc}
\hline After & 8 weeks & 18 weeks \\
\hline Ethanol intake & g/kg/day & g/kg/day \\
Total ethanol & $7.35 \pm 0.44$ & $7.80 \pm 0.5$ \\
$10 \%$ ethanol & $6.36 \pm 0.42$ & $6.00 \pm 0.31$ \\
$20 \%$ ethanol & $0.99 \pm 0.19$ & $1.36 \pm 0.50$ \\
Water intake & $\mathrm{ml} / \mathrm{kg} /$ day & $\mathrm{ml} / \mathrm{kg} /$ day \\
Water & $25 \pm 5$ & $20 \pm 8$ \\
Total water intake & $118.1 \pm 6.7$ & $116.6 \pm 7.8$ \\
\hline
\end{tabular}

ethanol free choice consumption and the last seven consecutive days after a period of 18 weeks of continuous ethanol consumption.

The non deprived group that was allowed to have 24-hr continuous access to ethanol during all the 18 weeks of the experiments never approached intakes as seen after 
each deprivation. Also, the base ethanol intake (g/kg/ day), the average of the 7 days immediately before deprivation did not change as a result of the deprivation periods. Data analyzed by one-way ANOVA indicate no significant difference in the total ethanol intake $[(\mathrm{F} 2,29)$ $=0.02 ; \mathrm{ns}] ; 10 \%$ ethanol intake $[(\mathrm{F} 2,29)=0.27$; ns $]$ and $20 \%$ ethanol intake $[(\mathrm{F} 2,29)=0.19$; ns] between base data.

\section{Discussion}

UChB rats consuming ethanol voluntarily for two months, exhibit a robust ADE after a single deprivation period of two weeks. The increased alcohol intake during the early days of re-exposure, following a withdrawal phase, is attributed to a shift in preference towards the higher concentration of ethanol that might reflect an increase in craving for alcohol, which returns to baseline after a period of two days.

Examining the effects of the second or third deprivation on ethanol intake, during the initial 24-hr period after the reinstatement of ethanol, the second and third deprivation cycle did not enhance the amount of ethanol intake above that was attained with the first deprivation. This could be the result of a ceiling effect (i.e. rats are drinking as much as possible under the experimental conditions). Repeated ADE evoked a drinking pattern in $\mathrm{UChB}$ rats very similar to that observed in $\mathrm{P}$ rats [8]. The increase in the amount of ethanol consumed was not the result of an indiscriminating polydipsia generated by the period of abstinence. The total fluid intake by rats (sum of water from ethanol solution plus water from the water tube) was not altered during all the period of the study. The effects observed after a single deprivation or repeated deprivations were not observed in the non deprived control group, suggesting that chronic exposure to ethanol alone is not sufficient to produce such a marked increase in ethanol intake or a shift in preference for higher concentrations of ethanol.

Uncontrolled alcohol drinking behavior can be assessed after prolonged drug self-administration in some individuals, and similar to those humans, some rats selectively bred for their high voluntary ethanol consumption can also develop addiction-like behaviours, suggesting that specific genetic factors are involved in the transition between high ethanol intake and development of an alcohol deprivation effect [12,13]. Therefore, the results suggest that neurobiological alterations may be occurring during time and these alterations result in a marked increased of ethanol consumption and to the development of uncontrolled alcohol-drinking behavior.

Another approach to evaluate the occurrence and the degree of addiction in laboratory animals are based on changes in alcohol intake by making drug seeking consistently resistant to interfering factors like concurrent choice of preferred flavours such as saccharin or sucrose [14]. This condition can also been observed in UChB rats. The placement of a third bottle containing a highly palatable $0.2 \%$ saccharin solution, decreased the ethanol intake in UChB rats that were exposed for a period of 7 days to a $10 \%$ ethanol and water under free choice condition, but not in UChB rats exposed to the $10 \%$ ethanol solution for a period of 60 days [15]. Moreover, it is known that addicts lost the ability to stop drinking and continue seeking drugs in spite of adverse environmental conditions. As a matter of fact, it has been previously observed that under chronic ethanol intake UChB rats leads to marked tolerance to the aversive effects of disulfiram and cyanamide on ethanol intake despite the presence of consistently high levels of blood acetaldehyde [16]. These results are in accord also to the notion that UChB rats develop uncontrolled alcohol-drinking behaviour, because of its resistance to the aversive effects produced by an increase in blood acetaldehyde levels.

There are several independent lines of evidence for genetic contributions to vulnerability to alcoholism [17]. Genetics is just beginning to illuminate the variations among individuals in their response to alcohol and one question is what are the changes in the brain that underlie the transition from controlled to uncontrolled alcohol use. According to Nestler $[18,19]$, repeated exposure to a drug of abuse alters the amounts, and even the types of genes expressed in specific brain regions resulting in alterations in molecular and cellular mechanisms that underlie long-term associative memories in several forebrain circuits. Whereas some alcoholics can cease drinking, others cannot. Despite multiple episodes of treatment, and despite risk of significant life problems, relapses to alcohol drinking is very frequent. As a matter of fact, the alcohol deprivation effect (ADE) has been proposed as an animal model of "loss of control" and "relapse-like” drinking [4,5]. Since an alcohol deprivation effect (ADE) is also observed in UChB rats make this line of rats selectively bred for their high voluntary ethanol consumption, a useful model for study the efficacy of pharmacological agents for the treatment of relapse of ethanol consumption.

\section{Conclusions}

Relapse to alcohol use after prolonged withdrawal periods is the major problem in the treatment of alcohol dependence in humans. However, there are relatively few studies concentrated on elucidation of the neurochemical events underlying relapse to alcohol. The ADE is a useful model for studying alcohol relapse in both rats and 
mice. Genetics, stress, and environmental cues are all important factors that influence relapse. Long-term alterations in neuronal activity within the glutamatergic and dopaminergic/serotoninergic systems, appears to underlie alcohol relapse.

\section{Acknowledgements}

We thank Mr. Juan Santibáñez for the expert care of the animals.

\section{References}

[1] R. Spanagel and S. M. Hölter, "Long-term Alcohol Self-administration with Repeated Alcohol Deprivation Phases: An Animal Model of Alcoholism?" Alcohol and Alcoholism, Vol. 34, No. 2, 1999, pp. 231-243. doi:10.1093/alcalc/34.2.231

[2] G. F. Koob and M. Le Moal, "Drug Addiction, Dysregulation of Reward, and Allostasis," Neuropsychopharmacology, Vol. 24, No. 2, 2001, pp. 97-129. doi:10.1016/S0893-133X(00)00195-0

[3] G. F. Koob, “Alcoholism: Allostasis and Beyond,” Alcoholism: Clinical and Experimental Research, Vol. 27, No. 2, 2003, pp. 232-243. doi:10.1097/01.ALC.0000057122.36127.C2

[4] R. L. Bell, Z. A. Rodd, J. A. Schultz, C. L. Peper, L. Lumeng, J. M. Murphy and W. J. McBride, "Effects of Short Deprivation and Re-exposure Intervals on the Ethanol Drinking Behavior of Selectively Bred High Alcohol-consuming Rats," Alcohol, Vol. 42, No. 5, 2008, pp. 407-416. doi:10.1016/j.alcohol.2008.03.130

[5] J. D. Sinclair and T. K. Li, "Long and Short Alcohol Deprivation: Effects on AA and P Alcohol-preferring Rats,” Alcohol, Vol. 6, No. 6, 1989, pp. 505-509. doi:10.1016/0741-8329(89)90059-1

[6] J. D. Sinclair and R. J. Senter, "Increased Preference for Ethanol in Rats Following Deprivation," Psychonomic Science, Vol. 8, 1967, pp. 11-12.

[7] Z. A. Rodd-Henricks, D. L. McKinzie, S. A. Shaikh, J. M. Murphy, W. J. McBride, L. Lumeng and T. K. Li, “Alcohol Deprivation Effect is Prolonged in the Alcohol Preferring (P) Rat after Repeated Deprivations,” Alcoholism: Clinical and Experimental Research, Vol. 24, No. 1, 2000, pp. 8-16. doi:10.1111/j.1530-0277.2000.tb04546.x

[8] Z. A. Rodd-Henricks, R. L. Bell, K. A. Kuc, J. M. Murphy, W. J. McBride, L. Lumeng and T. K. Li, "Effects of Concurrent Access to Multiple Ethanol Concentrations and Repeated Deprivations on Ethanol Intake of Alco- hol-preferring Rats," Alcoholism: Clinical and Experimental Research, Vol. 25, No. 8, 2001, pp. 1140-1150. doi:10.1111/j.1530-0277.2001.tb02328.x

[9] S. Serra, G. Brunetti, G. Vacca, C. Lobina, M. Carai, G. L. Gessa and G. C. Colombo, "Stable Preference for High Ethanol Concentrations Alter Alcohol Deprivation in Sardinian Alcohol-preferring (sP) Rats,” Alcohol, Vol. 29, No. 2, 2003, pp. 101-108. doi:10.1016/S0741-8329(03)00003-X

[10] J. Mardones and N. Segovia-Riquelme, "Thirty-two Years of Selection of Rats by Ethanol Preference: UChA and UChB Strains," Neurobehavioral Toxicology and Teratology, Vol. 5, 1984, pp. 171-178.

[11] M. E. Quintanilla, Y. Israel, A. Sapag and L. Tampier, "The UChA and UChB Rat Lines: Metabolic and Genetic Differences Influencing Ethanol Intake,” Addiction Biology, Vol. 11, No. 3-4, 2006, pp. 310-323. doi:10.1111/j.1369-1600.2006.00030.x

[12] J. C. Crabbe, “Genetic Contributions to Addiction,” Annual Review of Psychology, Vol. 53, 2002, pp. 435-462. doi:10.1146/annurev.psych.53.100901.135142

[13] M. J. Kreek, D. A. Nielsen, E. R. Butelman and K. S. LaForge, "Genetic Influences on Impulsivity, Risk-taking, Stress Responsivity and Vulnerability to Drug Abuse and Addiction," Nature Neuroscience, Vol. 8, No. 11, 2005, pp. 1450-1457. doi:10.1038/nn1583

[14] J. Wolffgramm and A. Heyne, "From Controlled Drug Intake to Loss of Control: The Irreversible Development of Drug Addiction in the Rat," Behavioural Brain Research, Vol. 70, No. 1, 1995, pp. 77-94. doi:10.1016/0166-4328(95)00131-C

[15] L. Tampier and M. E. Quintanilla, "Effect of Concurrent Saccharin Intake on Ethanol Consumption by High-alcohol-drinking (UChB) Rats,” Addiction Biology, Vol. 14, No. 3, 2009, pp. 276-282. doi:10.1111/j.1369-1600.2009.00147.x

[16] L. Tampier, M. E. Quintanilla and Y. Israel, “Tolerance to Disulfiram Induced by Chronic Alcohol Intake in the Rat," Alcoholism: Clinical and Experimental Research, Vol. 32, No. 6, 2008, pp. 937-941. doi:10.1111/j.1530-0277.2008.00658.X

[17] C. R. Cloninger, M. Bohman and S. Sigvardsson, "Inheritance of Alcohol Abuse. Cross-fostering Analysis of Adopted Men,” Archives of General Psychiatry, Vol. 38, No. 8, 1981, pp. 861-868.

[18] E. J. Nestler, "Molecular Mechanisms of Drug Addiction,” Neuropharmacology, Vol. 47, No. 1001, 2004, pp. 24-32. doi:10.1016/j.neuropharm.2004.06.031

[19] E. J. Nestler, "Is There a Common Molecular Pathway for Addiction," Nature Neuroscience, Vol. 8, No. 11, 2005, pp. 1445-1449. doi:10.1038/nn1578 